\title{
Development of Ag/GO Incorporated onto PES Membrane with Improved Anti-Fouling Property
}

\author{
Banele Vatsha ${ }^{1,2, *}$, Jane C. Ngila ${ }^{2}$ and Richard Moutloali ${ }^{1}$ \\ ${ }^{1}$ Advanced Materials Division, DST/Mintek Nanotechnology Innovation Centre, Mintek, South Africa \\ ${ }^{2}$ Department of Applied Chemistry, University of Johannesburg, Doornfonteion Campus, Johannesburg, \\ South Africa
}

\begin{abstract}
Graphene and its derivatives have got increasingly application interests emanating from its unique properties. This work reports silver-graphene oxide sheets (Ag-GO) composite synthesis and then incorporated into Polyethersulfone (PES) casting solution. The composite casting mixtures were cast via phase inversion method. Graphene and its derivatives were characterised by ATR/FTIR, Raman, XRD and TEM. The morphology and performance of the neat PES and composite PES membranes were characterised by SEM, AFM, CA, permeation flux, protein (BSA) rejection, antifouling and antibacterial tests. The composite membranes exhibited a slightly higher permeation flux and then gradual decreased compared to neat PES membranes. However, the antifouling tests revealed that the composite membranes with Ag particles showed a preferable antifouling performance. The antibacterial tests confirmed that the composite membranes exhibited a effective antibacterial performance against both gram-positive $(E$. coli) and gram-negative (S. Aureus) strains.
\end{abstract}

Keywords: Graphene derivativies, Polyethersulfone, silver particles, antibacterial effect.

\section{INTRODUCTION}

Increased attention has been drawn to polymer nanocomposite membranes preparation in many fields due to their unique multi-functional properties. Different incorporation routes of nanomaterials into polymer matrix have been adopted where it can be achieved by either ex-situ or in-situ methods [1]. In particular, water treatment systems have adopted nanocomposite membranes to mitigate severe water contamination of different effluent [2].

Membranes made from a wide range of polymers i.e. Polyethersulfone, Polyamide, Polyvinyldiflouride and cellulose acetate, to name a few, are prone to fouling tendency. However, polyethersulfone (PES) remains most outstanding membrane matrix for ultrafiltration types. This is attributed to its good chemical resistance, thermal stability and mechanical properties [3]. The hydrophobicity character of the membrane is one of the factors that contributes to severe fouling tendency. Consequently, membrane fouling causes flux decline and high maintenance costs. A lot of strategies have been developed to improve the properties of the polymer membrane over the past years. Among the methods, the strategy of doping nanomaterials to casting solution to prepare polymer-nanomaterial composite membranes is promising owing to its simple preparation steps $[4,5]$.

*Address correspondence to this author at the Advanced Materials Division, DST/Mintek Nanotechnology Innovation Centre, Mintek, South Africa; Tel/Fax: +27 11709 4386/4480; E-mail: badz.ufh@gmail.com
Unique properties of carbon nanomaterials are reported to impart improved permeability, mechanical strength, fouling resistance and thermal stability of the polymer membrane [6]. Subsequently, they tend to form clusters onto the polymer matrix due to dispersing problem. However, graphene oxide (GO) possesses strong interaction with the polymer chain which results ease dispersing onto polymer matrix. In addition to that, GO has hydrophilic functional groups known to improve the wetting properties of the hydrophobic polymer membrane [6]. Zinadini et al. [7] developed an antifouling PES-graphene oxide nanoplates mixed matrix membrane. They investigated the effect of the embedded GO nanosheet onto PES membrane surface chemistry (morphology and composition) and performance (pure water flux, dye removal and fouling parameters). They found that the water flux of the nanocomposite membranes significantly improved after addition of graphene oxide ( $0.5 \mathrm{wt} . \%)$ and had the best antibiofouling property with the highest mean pore radius, and porosity. Wang et al. [8] developed a green approach for controllable synthesis of silver nanomaterials on graphene oxide sheets using spontaneous reduction. They found that $\mathrm{GO}$ can act as substrates and reducing agents to yield silver nanoparticles, nanocubes and dendrites depending on the reaction time.

In this work, it was the primary goal to investigate the influence of GO nanosheets and silver-GO sheets composite on the structure of the prepared nanocomposite membranes. Graphene and its derivatives were characterised by TEM, XRD and 
ATR/FTIR. The effect of casting mixture composition on the performance of the nanocomposite membranes were studied by SEM, AFM, CA, permeation flux, protein (BSA) rejection and antibacterial tests.

\section{MATERIALS AND METHODS}

\subsection{Materials and Chemicals}

PES used as membrane material was supplied by Solvay polymers, n-methyl-2-pyrrolidone (NMP) and polyvinylpyrrolidone (PVP, K40) purchased from DLD scientific (Durban, South Africa) were used as a solvent and additive, respectively. High purity water freshly obtained from a Millipore system (Millipore, USA) was used as a non-solvent coagulation medium. Graphene sheets were used as received from Sigma Aldrich, South Africa. Graphite (Aldrich) was used to manufacture graphene oxide. Nitric $\left(\mathrm{HNO}_{3}\right.$, Aldrich), sulfuric acid $\left(\mathrm{H}_{2} \mathrm{SO}_{4}\right.$, Aldrich), potassium permanganate $\left(\mathrm{KMnO}_{4}\right.$, Aldrich) and hydrogen peroxide $\left(\mathrm{H}_{2} \mathrm{O}_{2}\right.$, Aldrich) were used to oxidize graphite to grapheme oxide for exfoliation.

\subsection{Procedures}

\subsubsection{Preparation of Ag-GO Nanocomposite}

Hydrophilic graphene oxide (GO) was synthesised by oxidizing graphite according to the modified Hummers method [8, 9, 10, 11]. Graphite flakes (0.12 g) were added to a mixture of concentrated $\mathrm{H}_{2} \mathrm{SO}_{4} / \mathrm{HNO}_{3}(6 \mathrm{~mL}: 0.132 \mathrm{~mL}$ ), and $\mathrm{KMnO} 4(0.72 \mathrm{~g})$ were gradually added to this mixture with stirring at 35$451 \mathrm{C}$ for $2 \mathrm{~h}$. The mixture was then heated to $100{ }^{\circ} \mathrm{C}$ and stirred for $30 \mathrm{~min}$ after water $(42 \mathrm{~mL})$ and $\mathrm{H}_{2} \mathrm{O}_{2}$ $(1.2 \mathrm{~mL})$ were added. On being cooled to room temperature, the mixture was centrifuged (Hanil Science Industrial Co., Ltd., Mega 17 R) at 13,000 rpm for $15 \mathrm{~min}$ to remove acidic supernatant from the mixture. Subsequently, the acidic supernatant was removed after centrifugation finished, and deionized water (DI water) was then added to dilute acidic remnant from graphite oxide. The mixture of graphite oxide pellet and DI water was vortex-stirred for $1 \mathrm{~min}$ to redisperse the pellet. This process which centrifugation and vortex alternatively were conducted was repeated until nearly neutral aqueous solution was obtained. The pellet, graphite oxide, was then added in NMP. Exfoliation of graphite oxide to $\mathrm{GO}$ was achieved by ultrasonication using a tip sonicator (Sonic VCX-750, Sonics \& Materials, Inc.) in ice water bath for $1 \mathrm{~h}$.

Further, the Ag-GO composite was synthesized using a method adopted from Wang et al. (2012) [8]. First, a fixed amount of $\mathrm{AgNO}_{3}$ was added to different weights of GO $(0.2,0.5$ and $1.0 \mathrm{wt}$. \%) in NMP solvent and the mixture stirred for 12 hours. The solutions made were deposited on a revelant sample holder and subsequently analysed by TEM. For instance, a drop of Ag-GO solutions was deposited onto a copper TEM grid and then dried at room temperature prior to analysis.

\subsubsection{Preparation of Ag@GO/PES Nanocomposite Membrane}

Membrane casting solutions containing PES (18 wt. $\%$ ) as membrane material, PVP (wt. \%) as pore forming agent, with the appropriate amount of $\mathrm{GO}$ and Ag-GO naomaterials were prepared in NMP solvent (Table 1) to produce homogeneous solution. This was attained by stirring the mixture for $12 \mathrm{~h}$ at a room temperature. The casting solutions were then

Table 1: Components of Composite Membrane (wt. \%)

\begin{tabular}{|c|c|c|c|c|c|}
\hline Membrane ID & PES (wt.\%) & PVP (wt.\%) & GO (wt.\%) & $\mathrm{AgNO}_{3}(\mathrm{wt} . \%)$ & NMP (wt.\%) \\
\hline MO & 18 & 2 & - & - & 80 \\
\hline M1 & 18 & 2 & 0.2 & - & 79.8 \\
\hline M2 & 18 & 2 & 0.2 & 0.5 & 79.3 \\
\hline M3 & 18 & 2 & 0.5 & - & 79.5 \\
\hline M4 & 18 & 2 & 0.5 & 0.5 & 79 \\
\hline M5 & 18 & 2 & 1.0 & - & 79 \\
\hline M6 & 18 & 2 & 1.0 & 0.5 & 78.5 \\
\hline
\end{tabular}




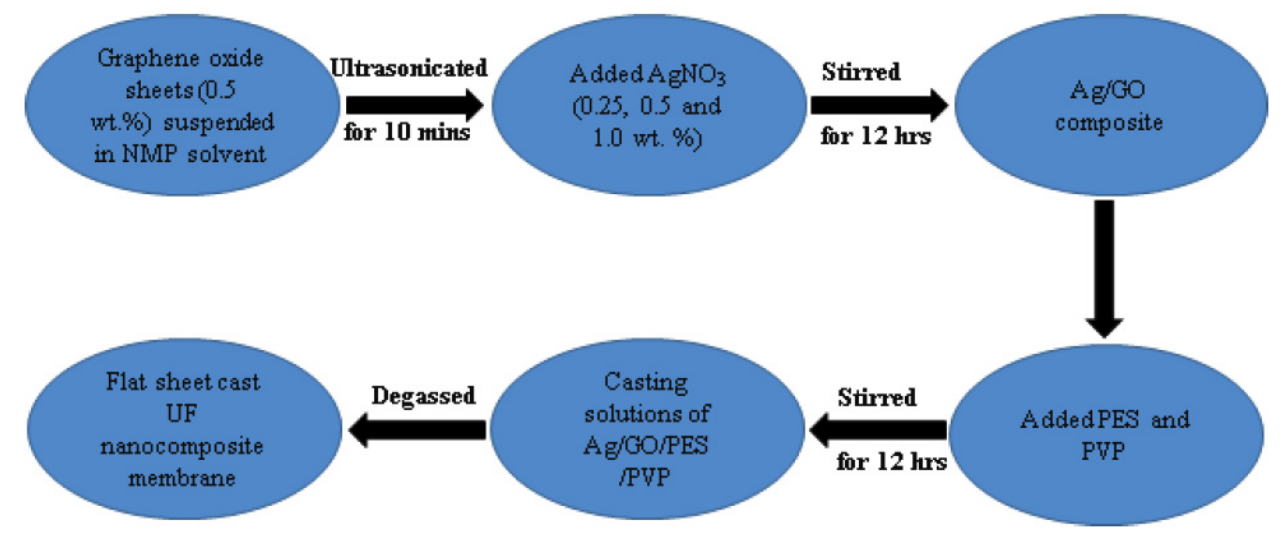

Scheme 1: Illustration of nanocomposites UF membrane preparation.

degassed using vacuum pump to allow a complete release of dissolved gases. The solutions were cast on glass plates using an automated Gardco knife, and then the glass plates were immersed in a coagulation bath of deionized water. All the membranes had a wet thickness of $\approx 250 \mu \mathrm{m}$ were peeled off and subsequently rinsed with water to remove the residual solvent and pore-forming agent. The resultant membranes were kept in water prior to ultrafiltration operation. The complete process from the preparation of $\mathrm{Ag}-\mathrm{GO}$ to membrane formation is captured in Scheme 1.

\subsection{Characterization Techniques Used}

\subsubsection{Transmission Electron Microscopy (TEM)}

The suspension solutions of all the samples were prepared in ethanol. A drop was placed in a copper grid and allowed to dry in a room temperature. TEM images were captured in a Philips CM120 Biotwin Transmission Electron Microscope (TEM). From the TEM images, it was easy to determine the sizes and shapes of the samples.

\subsubsection{Powder X-Ray Diffraction (XRD)}

Structural identification was performed using Powder X-ray Difrraction. The XRD diffractograms were recorded on a Bruker D8 Advance X-ray diffractometer using a Co-Ka (1.7902A $)$ monochromatic radiation source and a $\mathrm{Ni}$ filter with the operating voltage and current maintained at $35 \mathrm{kV}$ and $30 \mathrm{~mA}$, respectively, in the 2 theta range of $5^{\circ}-80^{\circ}$. The obtained diffraction patterns were exported and processed using OriginPro8 software.

\subsubsection{Fourier Transform-Infrared Spectroscopy (FT- IR)}

To observe the chemical structure changes of GO before and after modification, FT-IR/ATR spectra of pure $\mathrm{GO}$ and $\mathrm{Ag}$ doped $\mathrm{GO}$ (Ag-GO). All spectra were directly recorded on a Perkin Elmer spectrum 100 (MA, USA) Fourier Transform Infrared coupled with Attenuated Total Reflection (ATR-FTIR) spectrophotometer. All spectral were measured in wavenumbers in the IR range between $4000 \mathrm{~cm}^{-1}$ and $450 \mathrm{~cm}^{-1}$. A number of 64 scans were signal-averaged to minimise spectral noise.

\subsection{Membrane Characterization}

\subsubsection{Contact Angle Measurements}

Membranes contact angle values were measured on an Antension Theta system (Data physics, SCA20) following a procedure similar to that described by Shen et al. (2012) [12]. Where a needle tip was used to place a water drop $(5 \mu \mathrm{L})$ on the membrane surface. A digital camera was employed to magnify image of the droplet. Automated software was used to determine contact angle value. Five different points were collected on each membrane to yield contact angle value.

\subsubsection{Scanning Electron Microscopy (SEM)}

The dried membranes were mounted on a brass plate and then sputter coated with a thin film of carbon. Membrane surface images were obtained using CamScan SEM Model MV2300 microscope.

\subsubsection{Raman Spectroscopy}

The membrane surface chemistry was evaluated using a PerkinElmer RamanStation 400 bench-top, Raman spectrophotometer. The excitation source was a near-infrared $785 \mathrm{~nm}$ laser (100Mw at the sample), with a spot size of $100 \mu \mathrm{m}$. A spectral range of 220 $3200 \mathrm{~cm}$ was employed. The detector was a temperature controlled Charged Coupled Device (CCD) detector $\left(-50^{\circ} \mathrm{C}\right)$ incorporating a 1024 x 256 pixel sensor. Spectra were acquired using Spectrum 
software and images were acquired using Spectrum IMAGE software, both supplied by PerkinEImer (Bucks, UK).

\subsection{Filtration Experiments}

\subsubsection{Dead-End Filtration Cell System}

A dead-end filtration system with a $\mathrm{N}_{2}$ gas cylinder using pressure-driving force. This system is designed to evaluate the flat-sheet membrane performance. All UF membranes tests were performed using this system (Model 4750, Sterlitech purchased from USA) whose volume capacity was $400 \mathrm{~mL}$. The effective area of the membrane was $47.8 \mathrm{~m}^{2}$. The operation pressure in the system was maintained by nitrogen gas. Filtration tests i.e. permeation flux and protein BSA rejection were performed on the neat PES, PES-GO and PES/Ag-GO membranes. The membranes were pre-compacted for 10-15 minutes, before the permeate flux was recorded in every 5 minutes interval. Permeate flux $\left(J_{1}\right)$ was determined using equation (1.1).

$J_{1}=\frac{Q}{\Delta t \cdot A}$

The rejection was measured by filtrating BSA solution ( $1 \mathrm{~g} / \mathrm{L}, \mathrm{pH}=7.4$, PBS as buffer solution). After 60 minutes of filtration, the BSA concentrations in the feed solution and permeation solution were measured using UV-vis at $280 \mathrm{~nm}$ wavelength. The rejection (R) was calculated according to equation (1.2).

$R(\%)=\left(1-\frac{C_{p}}{C_{f}}\right) \times 100$

The cell was emptied and he fouled membrane was washed with high purity water for 20 minutes under shaking. The permeate flux $\left(J_{2}\right)$ was calculated from equation (1.3).

$J_{2}=\frac{Q}{\Delta t \cdot A}$

The flux recovery ratio (RF) was calculated according to equation (1.4).

$$
\operatorname{FRR}(\%)=\left(\frac{J_{2}}{J_{1}}\right)
$$

\subsection{Antibacterial Activity Tests}

Two cultural strains, i.e. E. coli and S. Aureus, were prepared by inoculating Luria Bertani (LB) Broth medium which was then incubated for $18 \mathrm{~h}$ at $37^{\circ} \mathrm{C}$ with shaking at $250 \mathrm{r} \cdot \mathrm{min}^{-1}$. Membranes and other glassware were sterilized by autoclaving for 15 minutes before use. Membranes were immersed into $5 \mathrm{ml}$ of culture containing approximately 106 colony forming units. $\mathrm{ml}^{-1}$ of bacteria. Membranes were allowed to soak in the culture solutions at room temperature for $24 \mathrm{~h}$, after which the membranes were retrieved and rinsed with $5 \mathrm{ml}$ sterile saline water. The wash solutions were then collected and diluted 10000 times $\left(10^{-4}\right)$ with deionized water, then aliquots $(100 \mu \mathrm{l})$ spread onto LB agar plates. Plates were incubated at $37^{\circ} \mathrm{C}$ for $24 \mathrm{~h}$, and number of colonies on the plates determined by the plate count method. Antibacterial rates were determined using equation 1.5 .

$R(\%)=\frac{m-n}{m} \times 100$

where $m$ and $n$ are the colony counts in neat PES, PES-GO and PES/Ag-GO composite membranes, respectively.

\section{RESULTS AND DISCUSSION}

\subsection{Ag-GO Composite Characterisation}

The crystal structures of $\mathrm{Ag}-\mathrm{G}, \mathrm{GO}$ and graphene were identified using X-ray diffraction. Scheme 2 depicts the synthesis protocol for Ag-GO composite and X-ray diffraction patterns (a). The XRD pattern of GO shows a strong peak located in the low-angle region $10^{\circ}$. The strong peak at $26.52^{\circ}$ in the pristine graphene pattern was not present in the graphite oxide pattern. In addition, new emerging peaking on Ag-GO are observed at $44^{\circ}, 52^{\circ}, 78^{\circ}$ and $93^{\circ}$.

TEM micrographs (Scheme 2: b, c and d) of the AgGO nanocomposites produced in this study are shown. The series of silver nanoparticles was deposited onto graphene oxide in NMP solvent under stirring. After aging the mixture for 12 hours, TEM analysis was used to confirm the uniform deposition of silver nanoparticles onto the surfaces of graphene oxide sheets. As the content of GO was increased at constant silver nitrate amount, an increase in the Ag nanoparticles deposited on the grapheme sheets was observed (Scheme 2). Wang et al. 2012 [8] previously elucidated that graphene oxide sheets simultaneously acts as the substrate and reducing agent for silver ions. They showed that the silver nanoparticles tend to predominately occupy both the terminal edges and steps formed by different sheets of the GO which are 
(a)
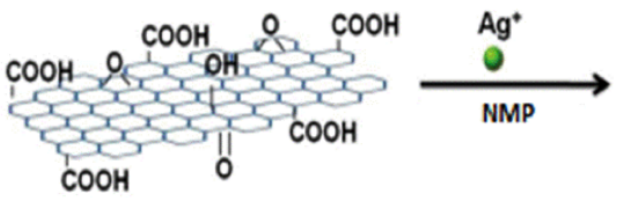
$\mathrm{COOH}$
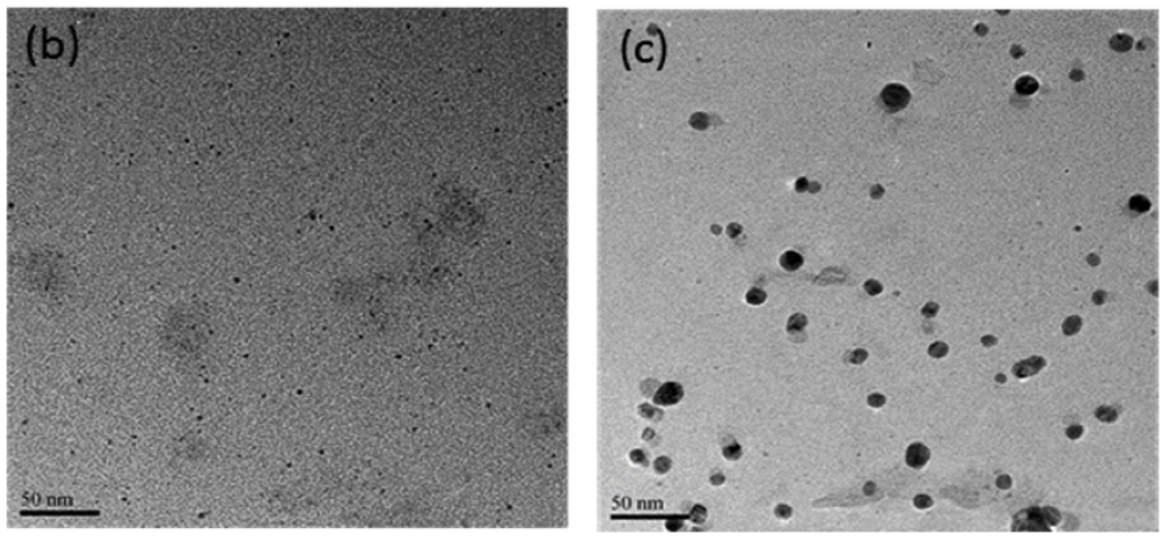

Scheme 2: Growth illustration of a) AgNPs onto graphene oxide sheets (Ag-GO) and XRD patterns for Ag-GO, GO, onset of Graphene. TEM micrographs of Ag-GO composite prepared from b) 1:2 ratio c) 1:1 ratio and d) 2:1 ratio [14, 15].

dominated by functional groups like carboxylic and carbonyl groups. Additional functional groups such as hydroxyl and epoxide groups, which are known to bind with silver nanoparticles weaker than carboxylic group, also occur at these sites [13]. TEM was utilised to examine the morphology, structure and size of the synthesised Ag nanoparticles deposited onto the GO sheets. It was observed that silver nanoparticles were well dispersed onto the surface of GO sheets and all have spherical shape with an average diameter of 5, 9 and $7 \mathrm{~nm}$ corresponding to Scheme $2 \mathrm{~b}, \mathrm{c}$ and $\mathrm{d}$, respectively.

Prior Ag-GO composite preparation, the successful preparation of GO nanosheets was confirmed by ATRFTIR spectroscopy. The spectrum of GO shown in Figure 1, exhibited characteristics bands at 1047 and $1215 \mathrm{~cm}^{-1}$, corresponding to the alkoxy and epoxy C-O bonds, respectively. The adsorption band at $1350 \mathrm{~cm}^{-1}$ corresponds to $\mathrm{O}-\mathrm{H}$ deformations in the $\mathrm{C}-\mathrm{OH}$ groups. The band at $1732 \mathrm{~cm}^{-1}$ resulted from $\mathrm{C}=\mathrm{O}$ stretching vibrations in the carboxylic groups. More prominent hump band between $3000-3700 \mathrm{~cm}^{-1}$ is assigned to $\mathrm{O}-\mathrm{H}$ stretching vibrations in the $\mathrm{C}-\mathrm{OH}$ groups. A band at $1620 \mathrm{~cm}^{-1}$, attributed to the $\mathrm{sp}^{2}$ hybridized $\mathrm{C}=\mathrm{C}$ groups in the GO structure. This observation is in agreement with literature [16]. The significant decrease in the intensity of the characteristic peaks of the oxygenated functional groups for the Ag-GO sample (in
Figure 2) was due probably to the existence of silver nanoparticles (AgNPs) on the surface of GO.

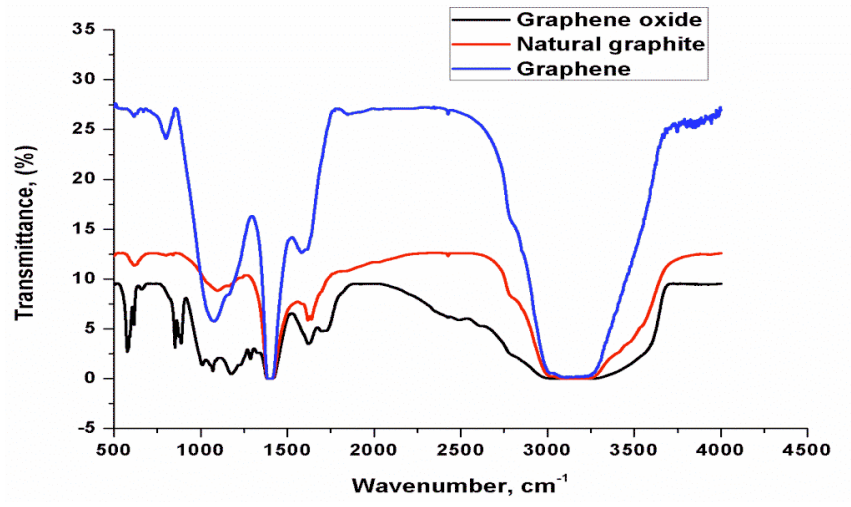

Figure 1: ATR/ FTIR spectra of natural graphite (red), graphene (blue) and graphene oxide (black) sheets.

\subsection{Membrane Characterisation}

\subsubsection{ATR Spectral Analysis of PES/Ag-GO Composite Membrane}

FTIR-ATR was used to examine membrane functional groups. The recorded spectra for the neat PES, PES-GO and PES/Ag-GO membranes are shown in Figure 2. The new emerging bands in the spectra of the composite membranes were observed at 3125 $\mathrm{cm}^{-1}, 1680 \mathrm{~cm}^{-1}$ and $1350 \mathrm{~cm}^{-1}$, which are attributed GO loading. neat PES membrane characteristic peaks 
were obtained. The peak at around $1295 \mathrm{~cm}^{-1}$ is arising due to $\mathrm{O}=\mathrm{S}=\mathrm{O}$ asymmetric stretching, where as the symmetric stretching of $\mathrm{O}=\mathrm{S}=\mathrm{O}$ giving a band at around $1150 \mathrm{~cm}^{-1}$. Band at around $1240 \mathrm{~cm}^{-1}$ and $1040 \mathrm{~cm}^{-1}$ are characteristic peaks corresponding to asymmetric and symmetric stretching vibrations of CO-C group. Also peaks between $2900 \mathrm{~cm}^{-1}$ and 3100 $\mathrm{cm}^{-1}$ correspond to aromatic and aliphatic $\mathrm{C}-\mathrm{H}$ stretching vibrations [17]. These observations further confirm the dispersion of $\mathrm{GO}$ in polyethersulfone matrix.

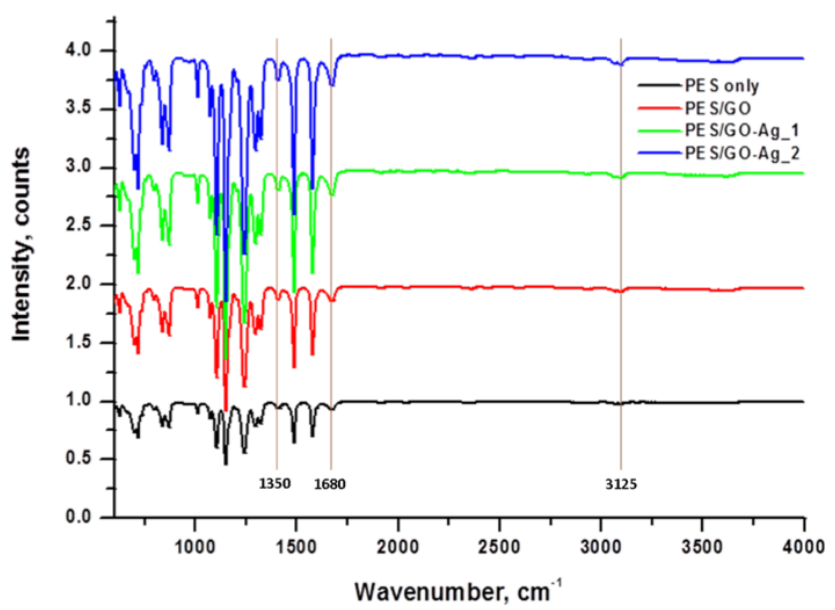

Figure 2: ATR/FTIR spectra of the neat PES, PES-GO and PES/Ag-GO membranes.

The synthesised nanocomposite PES-GO membrane matrix was further confirmed by Raman spectroscopy (In Figure 3). This technique is normally employed to elucidate the surface chemistry of carbonaceous materials i.e. graphene and carbon nanotubes. The most prominent Raman characteristic peaks of carbon materials are the $G$ and $D$ bands $\left(1580 \mathrm{~cm}^{-1}\right.$ and $\left.1350 \mathrm{~cm}^{-1}\right)$ which respectively represent

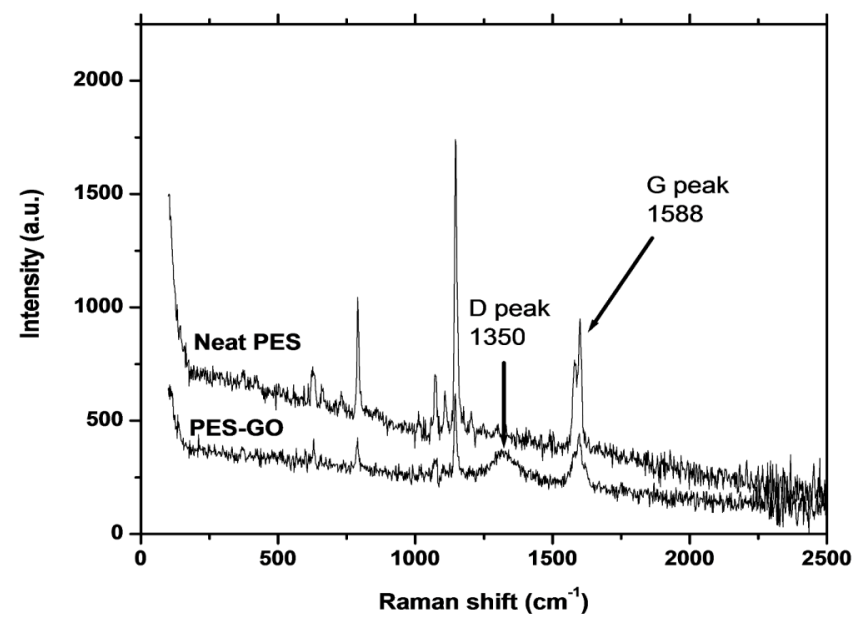

Figure 3: Raman spectra of neat PES and PES-GO nanocomposite membrane. the graphitized structure and local defects/disorders particularly located at the edges of graphene and graphite platelets $[14,15]$. The presence of AgNPs on graphene oxide sheets were confirmed by new pronounced $\mathrm{D}$ peak intensity compared to neat PES membrane. Thus, this mechanism has been wellexplored in the Raman surface enhancement studies.

\subsubsection{Morphological Observations of PES/Ag-GO Composite Membrane}

The SEM images of the neat PES and composite PES membranes are presented in Figure 4a-g. The results indicate that all the prepared membranes are composed of macroporous surface structure. This resulted from the presence of $\mathrm{GO}$ onto the polymer matrix. The SEM pictures in Figure 4a without GO depicts less surface pores. However, GO nanosheets presence onto the polymer matrix remarkable increased the membrane surface pores. At some point, when the GO content surpassed 0.20 wt. \% (Figure 4b and $\mathbf{c}$ ), yield a reduced membrane surface pores. This is ascribed to increased viscosity of the casting solutions with increased $\mathrm{GO}$ content, which suppresses formation of membrane macropores. Above this GO loading the membranes had a slightly dense structure with corresponding the pore size decrease (Figure 4cg).

A cross-sectional view of SEM micrographs of neat PES and composite PES/Ag-GO membranes are shown in Figure 5a-g. It was observed that all the synthesised membranes are made of asymmetric porous structure with a top skin layer and a finger-like porous sub-layer. The finger-like pores for all of the composite membranes (in Figure $\mathbf{5 b} \mathbf{b} \mathbf{g}$ ) are much wider than the neat PES membrane (in Figure 5a). This occurs due to the presence of $G O$ sheets in the composite membrane preparation step. The effect of high affinity $\mathrm{GO}$ sheets during phase inversion process improved the exchange rate of the solvent and nonsolvent components. This rapid mass transfer between the solvent and non-solvent components has therefore yielded more porous structure [18]. However, above GO (0.2 wt. \%) loading, lateral pore structures appeared within the PES/Ag-GO composite membranes changed from finger-like to sponge-like structures.

\subsubsection{Topographical AFM Analysis}

The surface AFM images of neat PES, PES-GO and PES/Ag-GO membranes are presented in Figure 6a-g. In the topographic images, the highest point of the membrane surface is represented by the brightest 

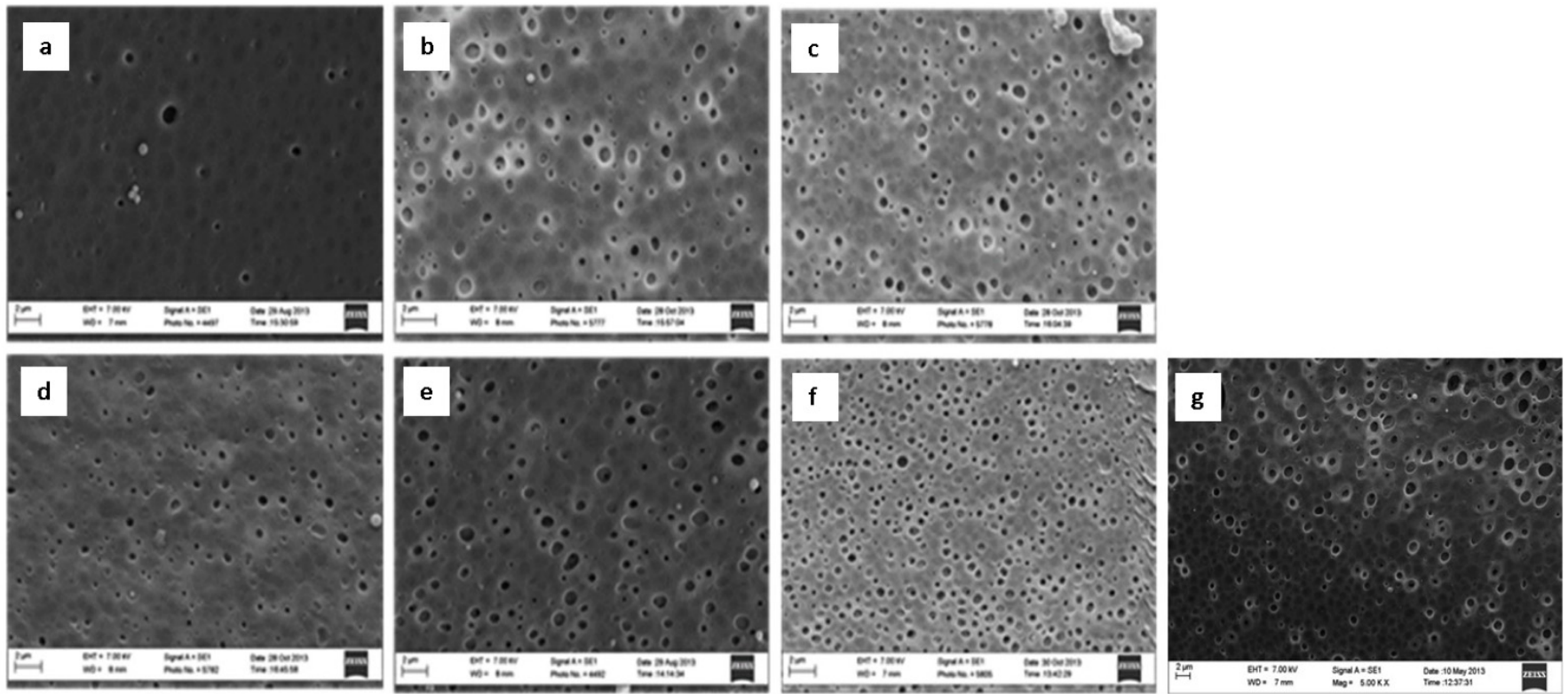

Figure 4: SEM micrographs of membrane's surface a) M0 b) M1 c) M2 d) M3 e) M4 f) M5, g) M6.
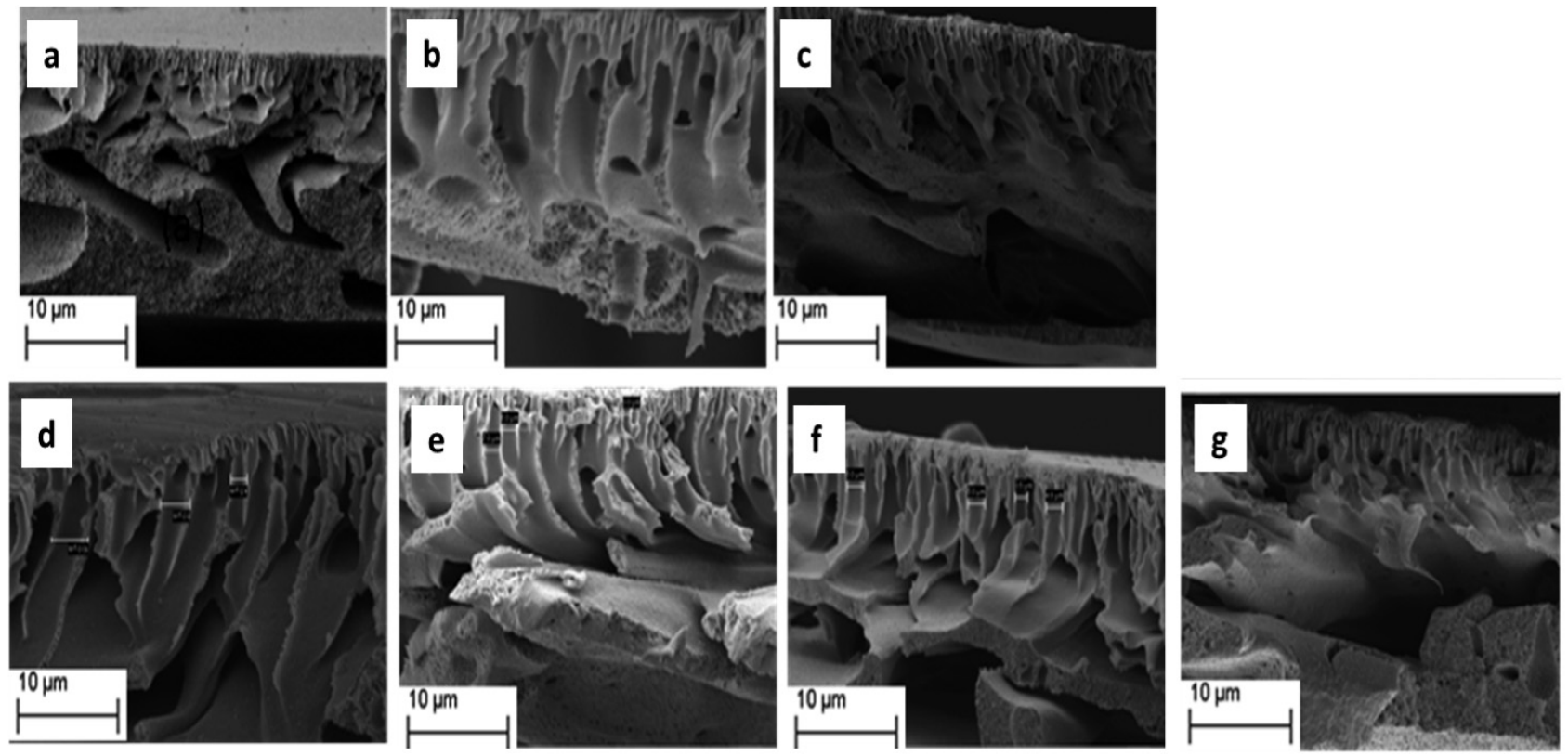

Figure 5: SEM cross-sectional micrographs of membrane's surface a) M0 b) M1 c) M2 d) M3 e) M4 f) M5 g) M6.

areas. The dark areas on the membrane surface are indicative of surface pores. In AFM images, increased surface pore sizes of the composite PES membranes compared to neat PES membrane were observed. However, at higher GO loadings, the number of surface pores decreased.

AFM images of the cast from neat PES, PES-GO and PES/Ag-GO membranes are shown in Figure 7a-g and the values of the surface roughness of the membranes are presented in Table 2. It is well known that the roughness values depend on the scan range. In this analysis, the scan range of $10 \mu \mathrm{m} \times 10 \mu \mathrm{m}$ was used and the RMS roughness of the neat PES is 20.4 $\mathrm{nm}$, while the RMS roughness of the composite membranes decreased to $9.7 \mathrm{~nm}$. However, at higher Ag-GO loading, the RMS roughness increased again. These values were similar to the literature. Zhang et al. [19] studied with composite PES-GO membranes and found that presence of $\mathrm{GO}$ nanosheets apparently lowered surface roughness.

\subsubsection{Contact Angle (CA) Measurement}

Membrane surface hydrophilicity/wettability is measured by contact angle (CA) values. It is known 

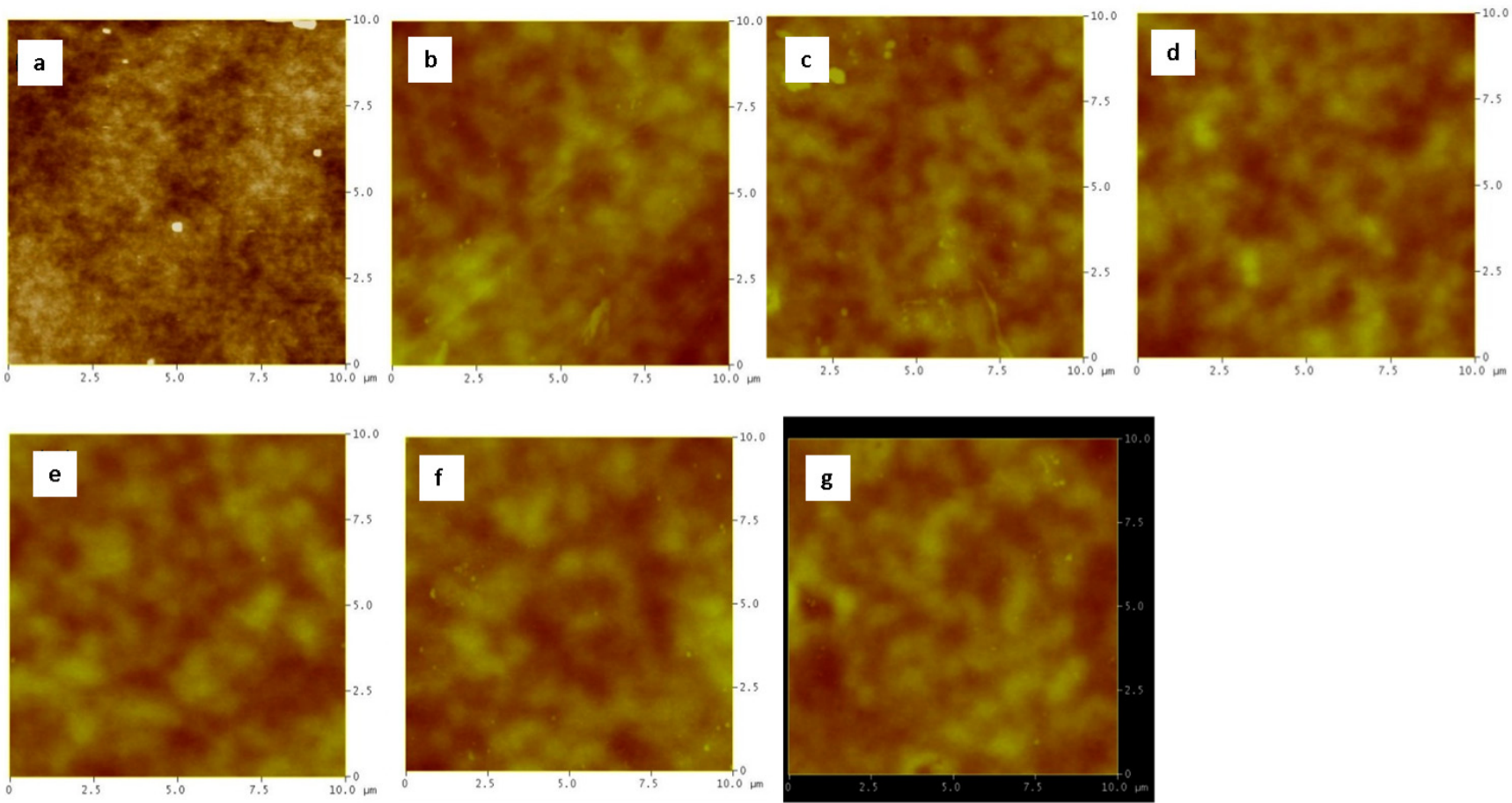

Figure 6: Membrane's topographic surface a) M0 b) M1, c) M2, d) M3, e) M4, f) M5, g) M6.
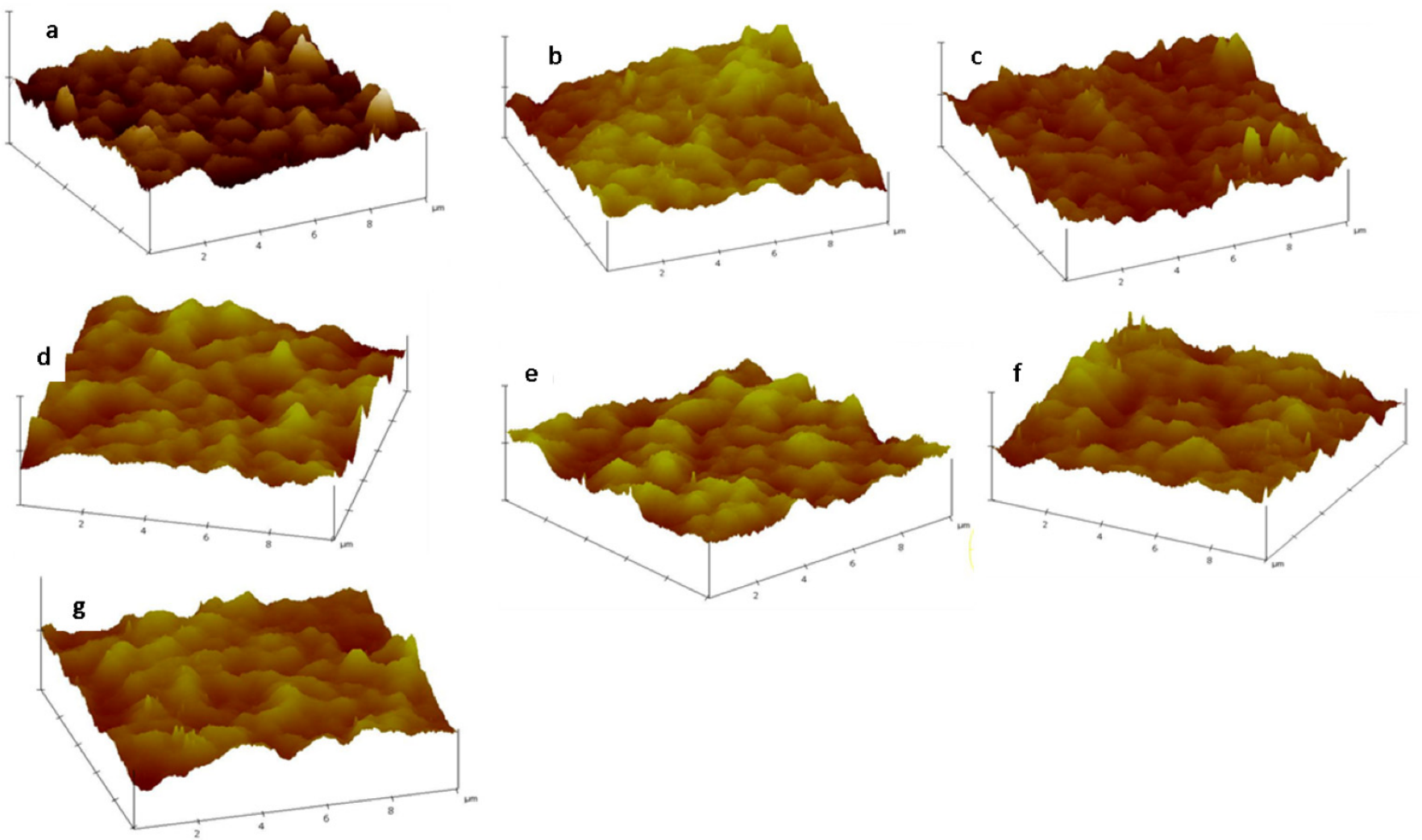

Figure 7: 3D AFM images of membrane's surface a) M0 b) M1, c) M2, d) M3, e) M4, f) M5, g) M5.

that the hydrophilic material exhibits smaller CA values. The CA measured immediately after water droplets were placed on the membrane surface, would preferably reflect the natural wettability of the material
[19]. The contact angle values of neat and composite PES membranes are given in Table 2. The neat PES membrane showed the highest water contact angle of $70^{\circ}$, whereas composites PES/GO and PES/Ag-GO 
membranes showed a remarkable decrease in water contact angle of $62^{\circ}$ and 54 , respectively ${ }^{\circ}$. This is indicative that with the presence of GO onto membrane surfaces promoted more hydrophilic character. This observation occurs when the membrane film solidifies during phase inversion process. A hydrophilic GO molecules migrates to the membrane/water interface [20]. In addition to that, authors have described that the presence of $\mathrm{Ag}$ particles on the composite membrane surface contributes to hydrophilic character.

Table 2: The Roughness and Contact Angle Values of Neat PES and Composite PES Membrane Surfaces

\begin{tabular}{|c|c|c|}
\hline Membrane ID & Roughness (nm) & Contact angle $\left({ }^{\circ} \mathbf{C}\right)$ \\
\hline \hline M0 & 20.4 & $70 \pm 1.2$ \\
\hline M1 & 9.7 & $62 \pm 1.5$ \\
\hline M2 & 10.1 & $64 \pm 1.7$ \\
\hline M3 & 15.3 & $60 \pm 2.4$ \\
\hline M4 & 14.2 & $61 \pm 3.5$ \\
\hline M5 & 18.2 & $56 \pm 2.7$ \\
\hline M6 & 18.6 & $54 \pm 2.2$ \\
\hline
\end{tabular}

\subsubsection{Flux and Rejection of PES/Ag-GO Composite Membrane}

Figure 8 presents the pure water flux of the neat PES membrane and composite PES/Ag-GO membranes. Herein it is observed that the pure water fluxes of the neat PES membrane is $170 \mathrm{~L} / \mathrm{m}^{2} . \mathrm{h}$ and composite membranes gradually increased to 246 $\mathrm{L} / \mathrm{m}^{2}$.h. This is achieved by the increased hydrophilicity character of the composite membranes due to the presence of Ag-GO composite. Authors indicated that GO acts as pore forming agent during phase inversion process. Sotto et al. (2011) [21] demonstrated that the water fluxes and contact angle of membranes have a reverse proportionality, this trend was also observed. Zang et al., (2013) [22] found that the addition of oxidized low-dimensional carbon nanomaterials into PES membrane matrix improve membrane structure (thermal and mechanical), morphology (pore size and porosity variations) and performance (flux and selectivity). Moreover, membrane porosity variation was obtained after Ag-GO nanocomposite addition. An Increase in membrane surface pores and improved hydrophilicity in the composite membrane structure resulted in higher water fluxes compared to the neat PES membrane. It is assumed that addition of nanoparticles in the casting solution altered the interaction between the polymer and the solvent components. This could be probably the reason for the formation of the macropores due to enhanced exchange rate of solvent and non-solvent components [18].

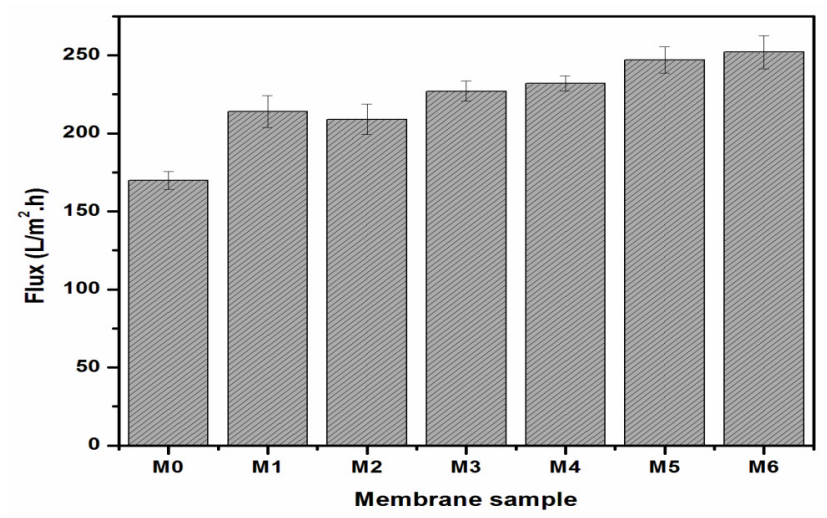

Figure 8: Water fluxes of the neat PES, PES-GO and PES/Ag-GO membranes at different loadings.

The flux decline during BSA rejection is observed compared to the pure water fluxes. This phenomenon occurs mainly due to the attachment of protein molecules on the membrane's surface. This can further cause the pores clogging/blockage on the membrane's surface. The protein rejections of the composite membranes were almost the same. BSA rejections of $96 \%$ above were observed for all the prepared membranes (Figure 9).

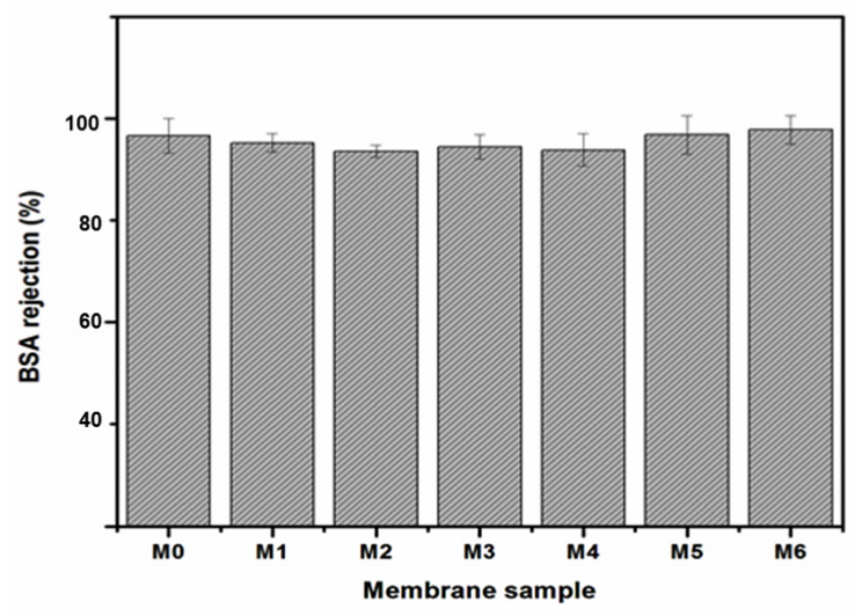

Figure 9: BSA rejection of the neat PES, PES-GO and PES/Ag-GO membranes at different loadings.

The fouling resistance ratio (FRR) is the method used to evaluate the membrane's antifouling property. The FRR (\%) values of the prepared neat and composite membranes increased as shown in Figure 10. This trend confirms that the induced hydrophilic GO improved the composite membrane's antifouling property. The increased hydrophilic groups on the 
membrane surface resulted in a strong interaction with water molecules. The attachment of BSA protein molecules on the membrane's surface was minimised.

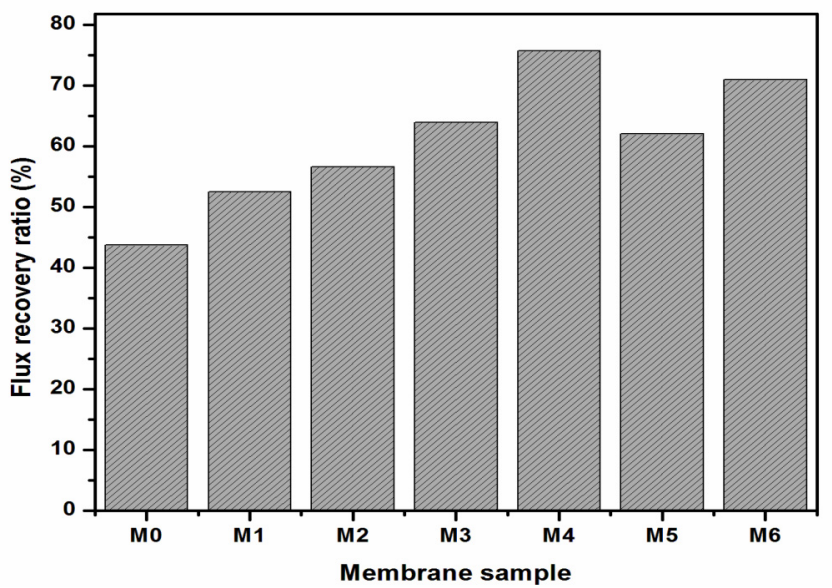

Figure 10: Effect of the GO content on flux recovery ratio.

\subsection{Antibacterial Tests of Membranes}

Antibacterial activities of neat PES, PES-GO and PES/Ag-GO membranes against E. Coli (Table 3) and S. Aureus (Table 4).

The antimicrobial activity of a polyethersulfone (PES) membrane doped with graphene oxide and silver nanoparticles was tested using E. coli and S. Aureus as the models of the Gram-negative and the Grampositive bacteria, respectively. This was done by comparing the disinfection rates of a PES membrane containing graphene oxide (GO) and silver nanoparticles grown on the surface of graphene oxide (Ag-GO), to that of a neat PES membrane, using the viable cell count technique. As shown in Figure 11a-c, the number of colonies on the plates treated with neat
PES membranes (a) is much higher than that on plates treated with PES- GO (b) and PES/Ag-GO (c) membranes. Furthermore, the colony count from plates treated with PES/Ag-GO membranes (c) is much less compared to other plates. As reported in the literature, the antibacterial activity of PES-GO and PES/Ag-GO was higher in E. coli (gram-ve) compared to that of $S$. aureus (gram +ve), with disinfection rates of $70.99 \%$ (PES-GO) and $95.85 \%$ (PES/Ag-GO). The increase in the disinfection rate of PES/Ag-GO membrane is partly due to the synergistic effects of silver and graphene oxide nanoparticles which have been reported to exhibit colossal antibacterial activities. These observations show that the presence of $\mathrm{Ag} / \mathrm{GO}$ on the PES membrane surface prevents the attachment of particulates or bacteria growth. In so doing, these composite membranes demonstrate a good anti-fouling property.

\section{CONCLUSIONS}

Ag-GO nanocomposite was successfully prepared and characterised by TEM and FTIR/ATR techniques. The Ag-GO nanocomposite based PES membranes were cast by the phase inversion method. The distribution of $\mathrm{Ag}$ doped $\mathrm{GO}$ nano-sheets were observed in PES membrane matrix. The TEM diameter of the prepared nanoparticles were measured to range between 5-15 nm. Smaller contact angle values were obtained implying the hydrophilicity improvement of the membranes. Therefore, the presence of $\mathrm{Ag}$ nanoparticles contributed to membrane antifouling properties. The antibacterial property of the neat PES and Ag-GO/PES membranes were evaluated using a halo zone test. Higher antibacterial property of the AgGO/PES membranes against $E$. coli and $S$. Aureus

Table 3: Antibacterial Activity of Membrane Against E. coli

\begin{tabular}{|c|c|c|}
\hline & No. of bacterial colonies (cfu) & Antibacterial rate (\%) \\
\hline \hline Pure PES membrane & 362 & 70.99 \\
\hline PES-GO & 105 & 95.85 \\
\hline PES/Ag-GO & 15 & - \\
\hline
\end{tabular}

Table 4: Antibacterial Activity of Membrane Against S. aureus

\begin{tabular}{|c|c|c|}
\hline & No. of bacterial colonies (cfu) & Antibacterial rate (\%) \\
\hline \hline Neat PES membrane & 397 & - \\
\hline PES-GO & 318 & 79.90 \\
\hline PES/Ag-GO & 89 & 77.52 \\
\hline
\end{tabular}




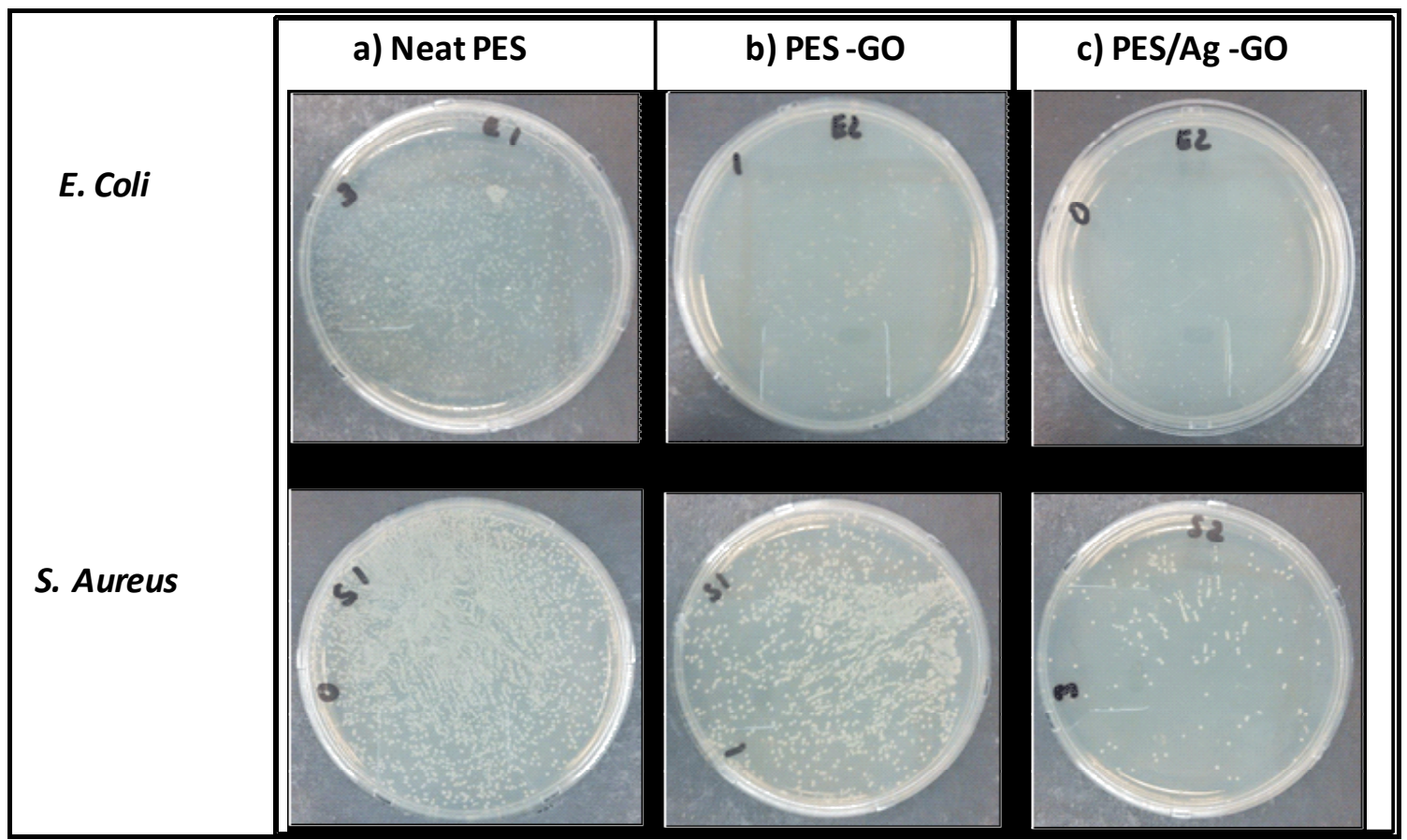

Figure 11: Colony counting measurement of antibacterial activity of neat PES and composite membranes using $E$. coli and S. aureus as model organisms. a) PES only (M1); b) PES-GO composite (M3) and c) PES/Ag-GO composite (M4).

were observed compared to neat PES and PES-GO membranes. This study observed that the Ag-GO/PES membranes can be useful for organic and biological fouling prevention in wastewater treatment systems.

\section{ACKNOWLEDGEMENTS}

The authors would like to thank DST-NRF for Professional Development Program (PDP) research grant through the DST/Mintek Nanotechnology Innovation Center platform.

\section{REFERENCES}

[1] Taurozzi JS, Arul H, Bosak, V. Z, Burban AF, Voice TC, Bruening ML, Tarabara VV. Effect of filler incorporation route on the properties of polysulfone-silver nanocomposite membranes of different porosities. J Mem Sci 2008; 325: 588.

http://dx.doi.org/10.1016/j.memsci.2008.07.010

[2] Zhang X, Cheng C, Zhao J, Ma L, Sun S, Zhao C. Polyethersulfone enwrapped graphene oxide porous particles for water treatment. Chem Eng J 2013; 215-216: 72-81. http://dx.doi.org/10.1016/j.cej.2012.11.009

[3] $\mathrm{Yu}$ L, Zhang $\mathrm{Y}$, Zhang B, Liu J, Zhang $\mathrm{H}$, Song C. Preparation and characterization of HPEI-GO/PES ultrafiltration membrane with antifouling and antibacterial properties. J Mem Sci 2013; 447: 452-462. http://dx.doi.org/10.1016/j.memsci.2013.07.042

[4] Zhang J, Xu Z, Shan M, Zhou B, Li Y, Li B, Niu J, Qian X. Synergetic effects of oxidized carbon nanotubes and graphene oxide on fouling control and anti-fouling mechanism of polyvinylidene fluoride ultrafiltration membranes. J Mem Sci 2013; 448: 81-92. http://dx.doi.org/10.1016/j.memsci.2013.07.064
[5] Zhang Y, Liu F, Lu Y, Zhao L, Song L. Investigation of phosphorylated $\mathrm{TiO}_{2}-\mathrm{SiO}_{2}$ particles/polysulfone composite membrane for wastewater treatment. Des 2013; 324: 118126. http://dx.doi.org/10.1016/j.desal.2013.06.007

[6] Jin F, Lv W, Zhang C, Li Z, Su R, Qi W, Yang QH, He Z. High-performance ultrafiltration membranes based on polyethersulfone-graphene oxide composites. RSC Adv 2013; 3(44): 21394-21397.

http://dx.doi.org/10.1039/c3ra42908c

[7] Zinadini S, Zinatizadeh AA, Rahimi M, Vatanpour V, Zangeneh $\mathrm{H}$. Preparation of a novel antifouling mixed matrix PES membrane by embedding graphene oxide nanoplates. J Mem Sci 2014; 453(0): 292-301.

http://dx.doi.org/10.1016/j.memsci.2013.10.070

[8] Wang X, Huang P, Feng L, He M, Guo S, Shen G, Cui D. Green controllable synthesis of silver nanomaterials on graphene oxide sheets via spontaneous reduction. RSC Adv 2012; 2(9): 3816-3822.

http://dx.doi.org/10.1039/c2ra00008c

[9] Wang Y, Zhang D, Bao Q, Wu J, Wan Y. Controlled drug release characteristics and enhanced antibacterial effect of graphene oxide-drug intercalated layered double hydroxide hybrid films. J Mat Chem 2012; 22(43): 23106-23113. http://dx.doi.org/10.1039/c2jm35144g

[10] Murphy S, Huang L, Kamat PV. Reduced Graphene OxideSilver Nanoparticle Composite as an Active SERS Material. J Phys Chem C 2013; 117(9): 4740-4747.

http://dx.doi.org/10.1021/jp3108528

[11] Tang J, Chen Q, Xu L, Zhang S, Feng L, Cheng L, Xu H, Liu $Z$, Peng R. Graphene Oxide-Silver Nanocomposite As a Highly Effective Antibacterial Agent with Species-Specific Mechanisms. ACS App Mat Int 2013; 5(9): 3867-3874. http://dx.doi.org/10.1021/am4005495

[12] Shen L, Bian X, Lu X, Shi L, Liu Z, Chen L, Hou Z, Fan K. Preparation and characterization of $\mathrm{ZnO} /$ polyethersulfone (PES) hybrid membranes. Des 2012; 293: 21-29. http://dx.doi.org/10.1016/j.desal.2012.02.019 
[13] $\mathrm{He} \mathrm{Y,} \mathrm{Cui} \mathrm{H}$. Synthesis of highly chemiluminescent graphene oxide/silver nanoparticle nano-composites and their analytical applications. J Mat Chem 2012; 22(18): 90869091.

http://dx.doi.org/10.1039/c2jm16028e

[14] Xu WP, Zhang LC, Li JP, Lu Y, Li HH, Ma YN, Wang WD, Yu $\mathrm{SH}$. Facile synthesis of silver@graphene oxide nanocomposites and their enhanced antibacterial properties. J Mat Chem 2011; 21(12): 4593-4597.

http://dx.doi.org/10.1039/c0jm03376f

[15] Akhavan O, Ghaderi E. Escherichia coli bacteria reduce graphene oxide to bactericidal graphene in a self-limiting manner. Car 2012; 50(5): 1853-1860. http://dx.doi.org/10.1016/j.carbon.2011.12.035

[16] Hu M, Mi B. Enabling Graphene Oxide Nanosheets as Water Separation Membranes. Env Sci Tech 2013; 47(8): 37153723. http://dx.doi.org/10.1021/es400571g

[17] Pham VH, Cuong TV, Hur SH, Oh E, Kim EJ, Shin EW, Chung JS. Chemical functionalization of graphene sheets by solvothermal reduction of a graphene oxide suspension in $\mathrm{N}$ methyl-2-pyrrolidone. J Mat Chem 2011; 21(10): 3371-3377. http://dx.doi.org/10.1039/C0JM02790A
[18] Wang Z, Yu H, Xia J, Zhang F, Li F, Xia Y, Li Y. Novel GOblended PVDF ultrafiltration membranes. Des 2012; 299(0): 50-54. http://dx.doi.org/10.1016/j.desal.2012.05.015

[19] Zhang X, Cheng C, Zhao J, Ma L, Sun S, Zhao C. Polyethersulfone enwrapped graphene oxide porous particles for water treatment. Chem Eng J 2013; 215: 72-81. http://dx.doi.org/10.1016/j.cej.2012.11.009

[20] Ganesh BM, Isloor AM, Ismail AF. Enhanced hydrophilicity and salt rejection study of graphene oxide-polysulfone mixed matrix membrane. Des 2013; 313(0): 199-207. http://dx.doi.org/10.1016/j.desal.2012.11.037

[21] Sotto A, Boromand A, Balta S, Kim J, Van der Bruggen, B. Doping of polyethersulfone nanofiltration membranes: antifouling effect observed at ultralow concentrations of $\mathrm{TiO}_{2}$ nanoparticles. J Mat Chem 2011; 21(28): 10311-10320. http://dx.doi.org/10.1039/c1jm11040c

[22] Zhang J, Xu Z, Mai W, Min C, Zhou B, Shan M, Li Y, Yang C, Wang Z, Qian X. Improved hydrophilicity, permeability, antifouling and mechanical performance of PVDF composite ultrafiltration membranes tailored by oxidized lowdimensional carbon nanomaterials. J Mat Chem A 2013; 1(9): 3101-3111.

http://dx.doi.org/10.1039/c2ta01415g 